ARTICLE

\title{
On the limitations in assessing stability of oxygen evolution catalysts using aqueous model electrochemical cells
}

Julius Knöppel (10 1,2四, Maximilian Möckl ${ }^{3}$, Daniel Escalera-López ${ }^{1}$, Kevin Stojanovski1,2, Markus Bierling ${ }^{1,2}$, Thomas Böhm ${ }^{1,2}$, Simon Thiele (1) ${ }^{1,2}$, Matthias Rzepka ${ }^{3} \&$ Serhiy Cherevko (1) ${ }^{1 凶}$

Recent research indicates a severe discrepancy between oxygen evolution reaction catalysts dissolution in aqueous model systems and membrane electrode assemblies. This questions the relevance of the widespread aqueous testing for real world application. In this study, we aim to determine the processes responsible for the dissolution discrepancy. Experimental parameters known to diverge in both systems are individually tested for their influence on dissolution of an Ir-based catalyst. Ir dissolution is studied in an aqueous model system, a scanning flow cell coupled to an inductively coupled plasma mass spectrometer. Real dissolution rates of the Ir OER catalyst in membrane electrode assemblies are measured with a specifically developed, dedicated setup. Overestimated acidity in the anode catalyst layer and stabilization over time in real devices are proposed as main contributors to the dissolution discrepancy. The results shown here lead to clear guidelines for anode electrocatalyst testing parameters to resemble realistic electrolyzer operating conditions.

\footnotetext{
${ }^{1}$ Forschungszentrum Jülich GmbH, Helmholtz Institute Erlangen-Nürnberg for Renewable Energy (IEK-11), Erlangen, Germany. ${ }^{2}$ Department of Chemical and Biological Engineering, Friedrich-Alexander-Universität Erlangen-Nürnberg, Erlangen, Germany. ${ }^{3}$ ZAE Bayern, Electrochemical Energy Storage,

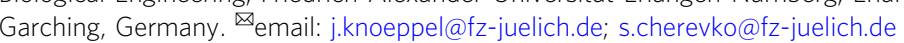


G lobal warming is driving the transition from fossil fuels to renewable energies. To support the transfer, economically promising alternatives to petrochemical processes for all sectors have to be established ${ }^{1}$. Owing to its high energy density, low chemical complexity, and high efficiency, hydrogen is among the best candidates for energy storage and distribution ${ }^{2-4}$. If hydrogen production from water electrolysis (WE) is fully supplied by renewable energy sources, greenhouse gas emissions can be reduced by $75 \% 5$. Therefore, research funding on upscaling WE technology is increasing ${ }^{6,7}$. Currently, technologies, based on liquid alkaline and acidic solid electrolyte are equally considered. Classical alkaline electrolyzers lack the option of dynamic operation necessary for direct coupling to fluctuating energy sources, and solid electrolyte anion exchange membrane (AEM) electrolyzers are not at a technology readiness level suitable for upscaling 8,9 . Acidic proton exchange membrane (PEM) electrolyzers consisting of membrane electrode assemblies (MEA), which lack these disadvantages, are the preferred system for upscaling in the short term ${ }^{10}$.

It is generally accepted that acidic conditions and high potentials at the anode side of PEM water electrolyzers (PEMWEs) where the oxygen evolution reaction (OER) takes place, demand for materials with high catalytic activity, and corrosion stability. Such criteria are only satisfied for electrocatalysts based on scarce noble metals such as iridium (Ir) and, to a lesser extent, ruthenium $(\mathrm{Ru})$. Although their current implementation in PEMWEs is not hampered by their cost, upscaling fabrication of MEAs with these metals is expected to be a major cost driver at the GW scale ${ }^{11,12}$. Therefore, a significant part of research activities on WE are focused on the reduction of noble metal content in PEMWE anodes ${ }^{13,14}$.

For the state-of-the-art Ir catalysts, a cornerstone in fundamental research studies has been to maximize Ir utilization, specifically, to increase their OER mass activity whilst reducing noble metal content without a significant loss in activity ${ }^{15,16}$. The use of high-surface-area catalyst supports ${ }^{17,18}$, highly active perovskites $^{19,20}$, and multimetallic materials ${ }^{21,22}$ are employed to reduce the noble metal content. However, stability has to be monitored as a second major descriptor in electrocatalyst design and synthesis, as OER catalysis also triggers catalyst dissolution ${ }^{23}$.

Activity and stability evaluations of newly developed catalysts are performed ex situ in the classical three-electrode electrochemical cell setup with acidic electrolyte to simulate the acidic $\mathrm{pH}$ environment of PEMWEs anodes in presence of Nafion ${ }^{24-26}$. Current-potential profiles are recorded and analyzed for activity evaluation. For stability evaluations, however, more sophisticated methods were developed, such as electrochemical quartz crystal microbalance ${ }^{27}$, scanning flow cell (SFC) coupled to an inductively coupled plasma mass spectrometer (SFC-ICP-MS $)^{28,29}$, and post-analysis of electrolyte and catalyst layers ${ }^{30,31}$. However, comparative data of catalyst stability in both systems show that degradation in aqueous systems does not represent the conditions in PEMWE ${ }^{32,33}$.

Recent results from our group, based on aqueous SFC-ICP-MS measurements and end-of life data from PEMWE, indicate an underestimation of the actual catalyst lifetime of several orders of magnitude, ranging from days in aqueous to years in $\mathrm{MEA}^{28}$. In this work, the relevance of S-numbers, a new metric for OER catalyst lifetime estimation, measured in aqueous systems for real application based on end-of-life data of PEM electrolyzers, is discussed. As end-of-life data is rarely found for Ir-based electrolyzers, the data set of a system using $\mathrm{RuO}_{2}$ as anode material published by Ayers et al. ${ }^{14}$ was compared with the same material measured in SFC-ICP-MS. It was found that the PEM electrolyzer outperforms the aqueous system by about three orders of magnitude, leading to a significant increase in the estimated lifetime of the electrolyzer in comparison to the same catalyst in the aqueous system. A similar concept, the activity-stability factor, was developed in parallel by Kim et al. ${ }^{34}$.

In this work, we aim to reveal the experimental factors responsible for the observed OER catalyst dissolution differences between aqueous model systems (AMS) and PEMWEs. We evaluated how the parameters that diverge between the systems such as catalyst loading, mass transport conditions, Nafion binder content, and electrolyte $\mathrm{pH}$ influence Ir dissolution. Also, we aim to determine the real dissolution rates of MEAs for PEMWEs using a custom-made full cell setup devised to prevent galvanic precipitation of catalyst dissolved species under OER operation.

\section{Results and discussion}

Iridium OER catalyst dissolution: aqueous model versus MEA systems. The dissolution behavior of OER catalysts in AMS is already well studied ${ }^{28,35-37}$. Utilizing online measurements, the dissolution behavior of OER catalysts under various electrochemical conditions has been shown. To put the results presented in this section into context, it is important to highlight the commonalities and differences between MEA and AMS. In AMS, the employed electrolyte, mostly an acid or base, is diluted by the reactant, deionized (DI) water. As schematically shown in Fig. 1a, the reaction products, $\mathrm{H}_{2}$ at the cathode side and $\mathrm{O}_{2}$ and protons at the anode side, as well as dissolution products, such as $\mathrm{Ir}^{3+}$,
Aqueous Model System

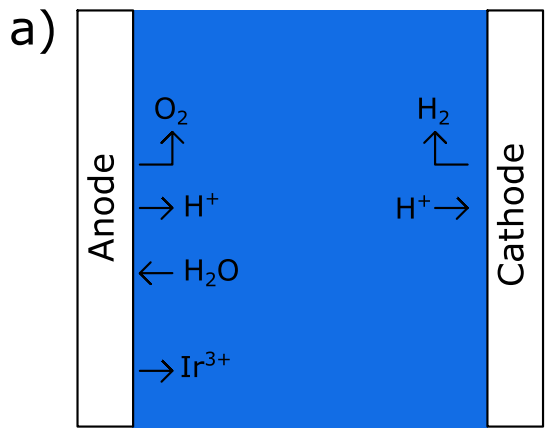

Membrane Electrode Assembly

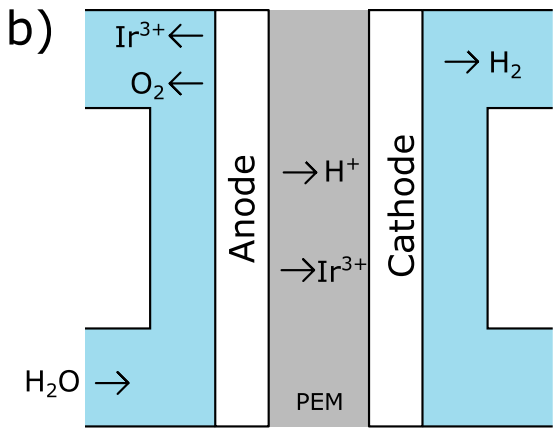

\section{Dissolution Comparison}

C)

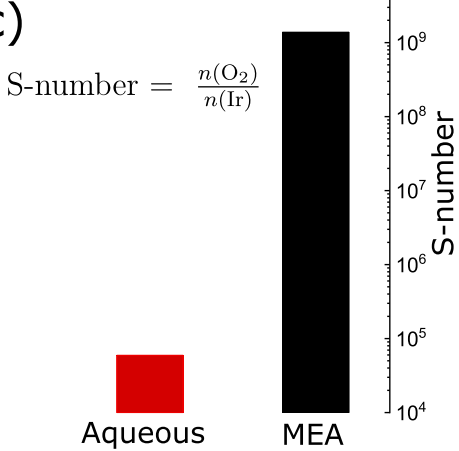

Fig. 1 Degradation processes of OER catalysts in aqueous and polymer electrolyte. a Schematic drawing of degradation processes in a classical aqueous electrolysis cell in aqueous electrolyte and $\mathbf{b}$ schematic drawing of degradation processes in MEA. c Dissolution stability of Ir $\mathrm{I}_{\mathrm{x}}$ under OER conditions in aqueous electrolyte, measured in SFC-ICP-MS, and polymer electrolyte, measured in a precipitation-free MEA device, expressed in the S-number metric. Measurements were carried out with a 5 min chronopotentiometry hold in aqueous electrolyte and over several days for MEA, typical timescales for the devices. Source data are provided in the source data file. 
diffuse into the bulk. Hence, to measure dissolution, it is sufficient to take aliquots of electrolyte from the solution. Utilizing flow cells, which directly transport reaction products from the reaction site downstream (if coupled) to analytical techniques such as ICPMS, the dissolution behavior can be directly correlated to the electrochemical operation $28,34,38$.

To study the degradation behavior of MEA, a system with a much higher degree of complexity, long-term measurements, and end-of-life (EOL) data have been used thus far. Owing to the long lifetime of MEA electrolyzers, however, EOL data are scarce. Furthermore, measurements of dissolution products in MEA are more complicated than in AMS. As schematically shown in Fig. 1b, electrolyte and reactant are decoupled in MEA by placing the polymer electrolyte between the electrodes and circulating DI water as the reactant at the backside. Reaction products, $\mathrm{H}_{2}$, and $\mathrm{O}_{2}$ escape through porous transport layers at the respective electrodes, whereas $\mathrm{H}^{+}$is transported through the PEM towards the reaction site at the cathode. In this system, dissolution products of OER catalysts have two ways to escape the anode catalyst layer: through the anode water cycle or the membrane towards the cathode side. Furthermore, galvanic replacement (GR) of dissolution products with stainless steel tubes, often employed in MEA test setups, can lead to an underestimation of dissolution $^{39}$.

Hence, to reliably determine dissolution in MEA and realistically compare results with AMS, several factors have to be controlled. The water level in the anode compartment and the water flow at the cathode outlet through electroosmotic drag have to be monitored at all times ${ }^{40,41}$. Furthermore, the amount of iridium depositing in the membrane has to be estimated. Also, GR should be excluded as a measurement factor.

For such purpose, a dedicated MEA setup without metallic parts in the anode water cycle was developed and employed in this study. Cell components involved in electronic conduction, namely titanium flowfields and current collectors, are coated with gold or platinum to prevent GR. Samples are taken from the anode water cycle and the cathode outlet and analyzed separately by ICP-MS. The setup and flow scheme is shown in supplementary note 1 . Water balance calculations, necessary to determine the amount of dissolved iridium, are shown in supplementary note 2.

To compare the dissolution stability of OER catalysts between MEA and AMS, a commercially available $\mathrm{IrO}_{\mathrm{x}}$ catalyst is measured in the aforementioned dedicated MEA system as well as in an SFC-ICP-MS setup operated with $0.1 \mathrm{M} \mathrm{H}_{2} \mathrm{SO}_{4}$. Figure $1 \mathrm{c}$ shows dissolution stability in both systems, displayed in the $\mathrm{S}$ number metric, a dimensionless descriptor that compares the amount of oxygen evolved, calculated from the measured current density at an estimated $100 \%$ faradaic efficiency towards OER, with the amount of iridium dissolved $\left(\mathrm{S}-\text { number }=\frac{n\left(\mathrm{O}_{2}\right)}{n(\mathrm{Ir})}\right)^{28} . \mathrm{S}$ numbers were calculated from constant current measurements of 5 minutes in AMS and several days in the MEA. Electrochemical data and dissolution data for MEA experiments are available in supplementary note 3 . Electrochemical data, dissolution data, and the integration areas for determining S-numbers in SFC-ICP-MS are shown in Fig. 2. Both timescales are representative of the respective system.

The S-number of $\mathrm{IrO}_{\mathrm{x}}$ in the SFC-ICP-MS is $6 \times 10^{4}$. Although with the used $\mathrm{H}_{2} \mathrm{SO}_{4}$ electrolyte, a stronger adsorption of anions on the surface is anticipated ${ }^{42}$, the measured $\mathrm{S}$-number is comparable to literature values measured in the noncoordinating $\mathrm{HClO}_{4}$, which range between $10^{4}$ and $10^{528,43-45}$. Thus, although the influence of the electrolyte anion cannot be fully ruled out, its role in the stability of $\mathrm{IrO}_{x}$ is minor. Remarkably, the observed S-number of $\mathrm{IrO}_{\mathrm{x}}$ in the MEA system

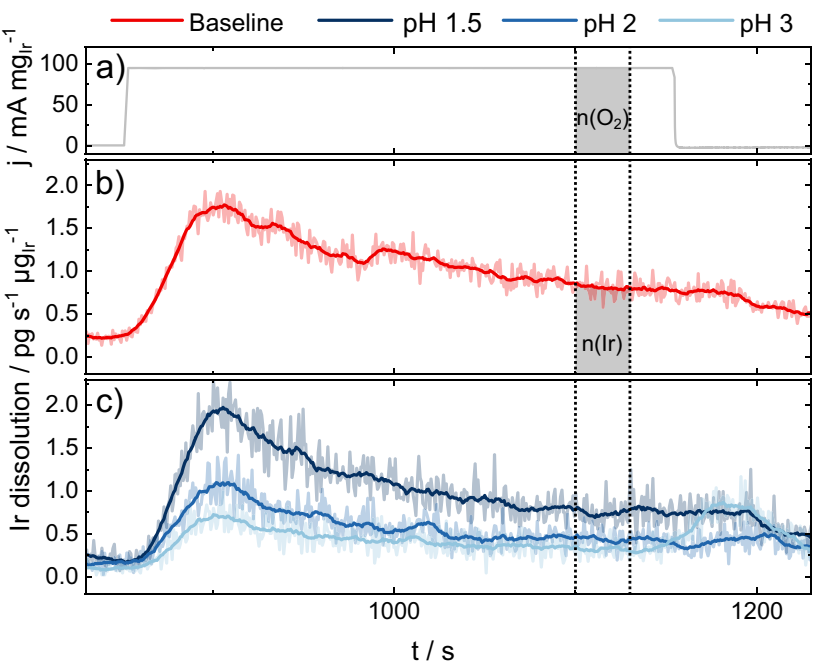

Fig. 2 Dissolution profiles of IrO $_{x}$ catalyst spots in SFC-ICP-MS

measurements. a Applied current step and $\mathbf{b}$, c resulting dissolution of $\mathbf{b}$ a baseline measurement at standard conditions $\left(10 \mu g_{\mathrm{Ir}} \mathrm{cm}^{-2}\right.$ catalyst loading,

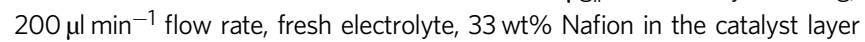
and $\left.0.1 \mathrm{M} \mathrm{H}_{2} \mathrm{SO}_{4}(\mathrm{pH}=1)\right)$ and $\mathbf{c}$ with a variation of electrolyte $\mathrm{pH}$. The integration area for the calculation of S-numbers is highlighted by vertical dashed lines. Source data are provided in the source data file.

exceeds the one observed in aqueous systems by almost five orders of magnitude. Further experiments in AMS were undertaken to unravel the reasons for this behavior.

Evaluation of model aqueous OER stability parameters. We evaluated several parameters that generally differ in both systems to determine the origin of the dissolution discrepancy between AMS and MEA. (a) catalyst loading; (b) electrolyte flow rate; (c) presence of electrochemically dissolved iridium species; (d) Nafion content in the catalyst layer; and (e) $\mathrm{pH}$ were individually varied during testing in the AMS (SFC-ICP-MS). All experiments were carried out with the same $\mathrm{IrO}_{\mathrm{x}}$ catalyst powder as in previously shown MEA experiments.

Dissolution profiles of baseline measurement of $\mathrm{IrO}_{\mathrm{x}}$ catalyst powder spots at a current step of $100 \mathrm{~mA} \mathrm{mg}_{\mathrm{Ir}}{ }^{-1}$ (Fig. 2a) are displayed in Fig. 2b. The full measurement protocol is shown in supplementary note 4 . Representative electrochemical and dissolution data for all experiments are shown in supplementary note 5.

The corresponding S-numbers for all experiments, determined at a 30 s steady-state interval at the end of the current step $^{28}$, as shown in Fig. 2a and b) are displayed in Fig. 3. All error bars are acquired from at least three independent measurements. The baseline measurement is hereby displayed in Fig. 3a).

Our first study focused on differences in catalyst loading. Although Ir loading in aqueous studies rarely exceeds $10 \mu \mathrm{g}_{\mathrm{Ir}} \mathrm{cm}^{-2}$, loading in MEA is typically $\sim 1-2 \mathrm{mg}_{\mathrm{Ir}} \mathrm{cm}^{-246}$. S-numbers of catalyst spots with different loading is shown in Fig. 3b, where loading is varied between $10 \mu \mathrm{g}_{\mathrm{Ir}} \mathrm{cm}^{-2}$ and $250 \mu \mathrm{g}_{\mathrm{Ir}} \mathrm{cm}^{-2}$. The obtained S-number values are comparable and in the same order of magnitude. Hence, we can exclude the influence of loading on the dissolution discrepancy.

The second study was different SFC operating flow rates. Given the flow rate uncertainty in PEMWE MEA systems, contrasting with its precise control in our SFC-ICP-MS setup, we evaluated the impact of SFC flow rate by variations within one order of magnitude. The flow rate of electrolyte to the ICP-MS was here varied between $66 \mu \mathrm{min}^{-1}$ and $740 \mu \mathrm{min}^{-1}$. S-numbers for these experiments are virtually equivalent as shown in Fig. 3c. As 


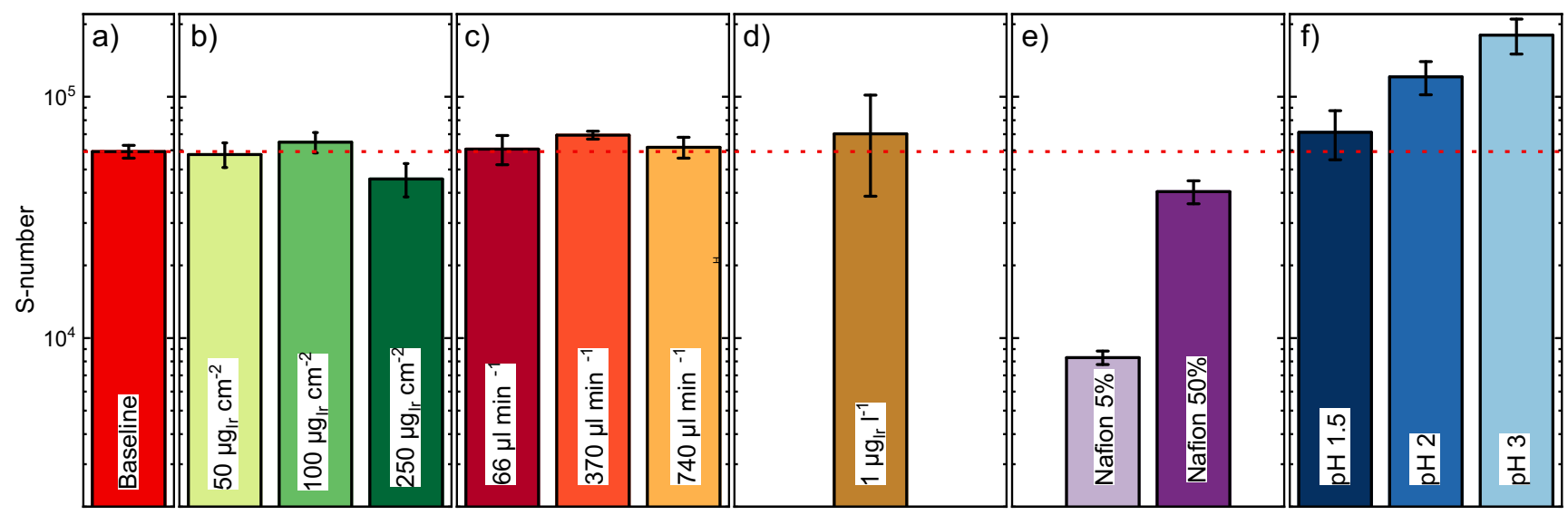

Fig. 3 S-numbers of $\mathbf{I r O}_{\mathbf{x}}$ catalyst spots with varied conditions, measured in SFC-ICP-MS. a Baseline measurement. $\mathbf{b}$ Variation of loading. $\mathbf{c}$ Variation of flow rate. $\mathbf{d}$ Addition of pre-dissolved iridium in the electrolyte. e Variation of Nafion content in the catalyst layer. $\mathbf{f}$ Variation of $\mathrm{pH}$. Red, dashed line:

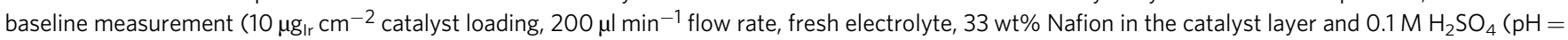
1)), displayed for ease of comparison between $\mathbf{b}$ and $\mathbf{f}$. Error bars were calculated as the standard deviation of at least three independent measurements. Source data are provided in the source data file.

the flow rate effectively dictates the mass transport rate of dissolved species from the electrode interface to the bulk electrolyte, a possible Ir redeposition mechanism should be affected by it as well. Based on the results obtained, these mass transport phenomena do not seem to play a key role in the AMSMEA catalyst stability differences.

The third parameter variation tackled the dissolution-redeposition equilibrium of Ir-dissolved species, which can occur during MEA water circulation owing to low flow rates and slow mass transport in thick catalyst layers. To simulate such conditions in AMS, and enable a potential dissolution-redeposition mechanism, electrochemically dissolved iridium has to be present in the electrolyte in relevant concentrations. Therefore, Ir was electrochemically dissolved from polycrystalline iridium ${ }^{37,47}$ (see the experimental section for further details) and then intentionally incorporated into the acidic electrolyte used. The iridium concentration in the electrolyte is determined by ICP-MS and adjusted accordingly. Figure 3d) shows the S-number of $\mathrm{IrO}_{\mathrm{x}}$ spots measured with $1 \mu \mathrm{g}_{\mathrm{Ir}} \mathrm{I}^{-1}$ dissolved iridium in the electrolyte. This value is similar to a recent publication, where Ir concentrations in MEAs were measured ${ }^{39}$. Also, it is in the same order of magnitude as the concentrations measured in our MEA study (see supplementary note 3 ). As observed with the previous parameters, S-number values are comparable to the baseline experiment. Hence, equilibrium states between dissolution and redeposition are unlikely to largely contribute to the Ir dissolution discrepancy.

The fourth evaluated parameter is the influence of Nafion content in the catalyst layer on Ir dissolution. Unlike OER rotating disk electrode experiments, where no Nafion is required, it acts as a catalyst layer binder in SFC-ICP-MS to avoid particle detachment. Figure $3 e$ shows the $\mathrm{S}$-numbers of $\mathrm{IrO}_{\mathrm{x}}$ spots with different Nafion contents, varied here between 5 and 50 wt $\%$ vs. the total catalyst content. Interestingly, the dissolution rate of catalysts spots with $5 \mathrm{wt} \%$ Nafion in the catalyst layer is significantly larger from the other Nafion contents. Indeed, the stability differs from baseline measurements by an order of magnitude, with an S-number of $8 \times 10^{3}$. We hypothesize that, at lower Nafion contents, dissolved iridium mass transport from the catalyst layer to the electrolyte bulk might be more efficient. A local saturation might hereby inhibit dissolution. As baseline measurements have a Nafion content of $33 \mathrm{wt} \%$, whereas MEA electrodes were produced with $9 \mathrm{wt} \%$ Nafion content in this study, Nafion content will have a role in stability observations.
However, different constraints have to be met for measurements in both systems. In SFC-ICP-MS, Nafion predominantly acts as a binder in the catalyst layer to avoid particle detachment. On the other hand, in the more complex MEA system, Nafion has not only an influence on the integrity of the catalyst layer, but also on features such as contact resistance with the porous transport layers (PTL). As Nafion contents employed in this study rather open the gap between AMS and MEA instead of closing it, this rather leads to an underestimation than an overestimation of the stability discrepancy.

The fifth study carried out is the variation of the working electrolyte $\mathrm{pH}$ value, varied here between 1 and 3 (experimental dissolution profiles shown in Fig. 2c). To ensure a similar ionic strength, a sulfate salt was added to electrolytes with $\mathrm{pH}>1$. The resulting S-numbers, shown in Fig. 3f), significantly differ from each other. Although the S-number metric obtained for $\mathrm{pH} 1.5$ is still similar to the reference measurement $(\mathrm{pH} 1)$, a significant difference is observed for $\mathrm{pH} 2$ and 3 . Such difference in stability is a factor of three: for $\mathrm{pH} 1, \mathrm{~S}$-number $=6 \times 10^{4}$ whilst for $\mathrm{pH}=$ 3 , S-number $=1.8 \times 10^{5}$. It should be noted that local $\mathrm{pH}$ at the electrode under OER conditions might be lower owing to the worsened buffer capacity of electrolytes with higher $\mathrm{pH}^{48}$.

MEA environments for PEMWE are, according to the literature, highly acidic owing to the use of Nafion ${ }^{49,50}$. The observations, made here in AMS, however, indicate, that the activity of protons in MEA electrolysis might be lower than generally accepted. Further experiments in MEA systems have to be conducted to unravel the magnitude of the differences.

Impact of pH in PEMWE operation: catalyst and MEA stability. As shown in a previous section, of all parameters evaluated in our study of model aqueous systems, $\mathrm{pH}$ is the only one with a relevant impact on stability towards closing the gap. Hence, measurements of $\mathrm{IrO}_{\mathrm{x}}$ in MEA were conducted with $0.1 \mathrm{M} \mathrm{H}_{2} \mathrm{SO}_{4}$ in the anode water cycle to investigate the influence of low $\mathrm{pH}$ environments on dissolution. The operational principles in both systems are schematically shown in Fig. 4a for MEA operated with DI water and in Fig. $4 \mathrm{~b}$ ) for the MEA operated with acid. $\mathrm{S}$-numbers from liquid samples collected after $2 \mathrm{~h}$ operation are displayed in Fig. 4c). Strikingly, the S-number of the conventional system exceeds the one from the acid operated by more than two orders of magnitude. In comparison to AMS, which operates with 

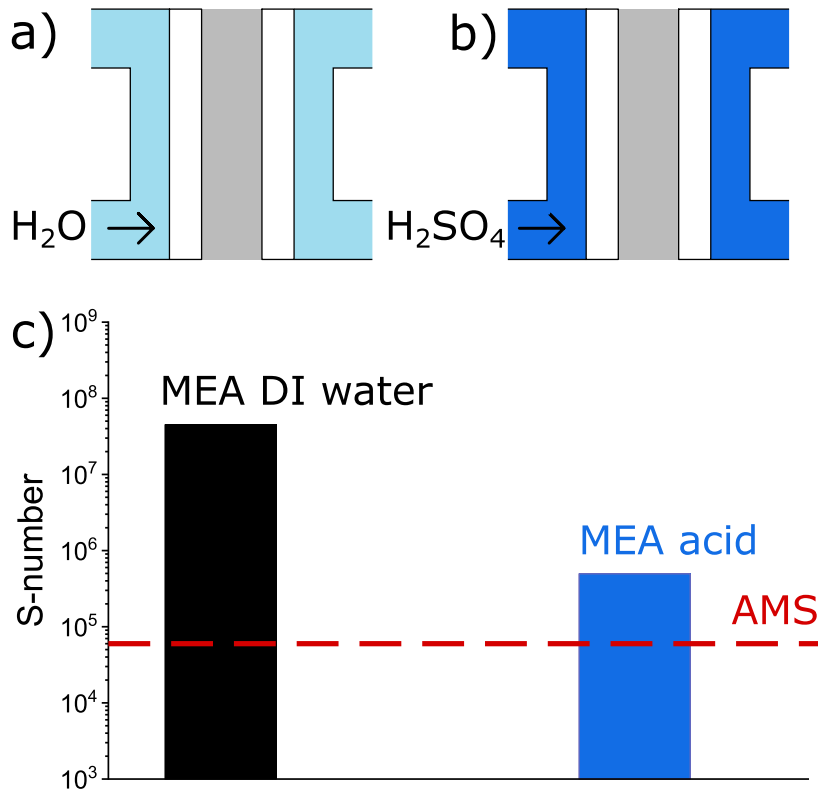

Fig. 4 Comparison of stability in different systems. a Working principle of an MEA operated with DI water. $\mathbf{b}$ Working principle of an MEA operated with $0.1 \mathrm{M} \mathrm{H}_{2} \mathrm{SO}_{4}$. c S-numbers of MEAs operated with DI water and $0.1 \mathrm{M}$ $\mathrm{H}_{2} \mathrm{SO}_{4}$ after $2 \mathrm{~h}$ of measurement. The red, dashed line in $\mathbf{c}$ indicates the $\mathrm{S}$ number of the AMS system. Source data are provided in the source data file.

S-numbers around $6 \times 10^{4}$, the stability difference to the acidic operated MEA virtually vanishes. Its impact can be easily grasped when calculating catalyst half-life estimated from S-numbers ${ }^{28}$. For a DI water-fed MEA system, its value is ca. 150 years, whereas for an acidified MEA it is just several days.

Post mortem scanning transmission electron microscopy (STEM) cross-section micrographs of the MEA after $48 \mathrm{~h}$ of continuous operation at $2 \mathrm{~A} \mathrm{mg}_{\mathrm{Ir}}{ }^{-1}$, shown in supplementary note 7 , reinforce the stark degradation differences found from liquid sample analysis. Although the anode catalyst layer of the DI water-operated MEA is virtually intact and iridium migration into the membrane is non-existent, the anode catalyst layer of the acidic operated MEA reveals exceptional signs of degradation. Indeed, $\mathrm{Au}$ particles, originating from the partly dissolved flowfield coating, of $\mu \mathrm{m}$ diameter form in the membrane close to the anode side. The cathode side of the conventional operated MEA only shows signals of $\mathrm{Pt}$ and $\mathrm{C}$, whereas iridium was detected in the catalyst layer of the acidified MEA. Furthermore, the MEA polarization curves indicate a shift in $\mathrm{pH}$ (for full description, see supplementary note 8$)^{51}$ and the anode flowfield and current collector displays stark signs of degradation after the experiment. (See supplementary note 9)

Impact of OER operating timescale in catalyst stability in AMS and MEA. The different timescales have to be taken into account when comparing AMS with MEA systems. For instance, the data shown in Fig. 1c were measured over minutes for AMS and days for MEA. Indeed, MEA systems have proven stable operation for thousands of hours on the laboratory $\mathrm{scal}^{52}$ and in industrial applications ${ }^{53}$. Hence, we investigated if the short experimental timescale in AMSs can be extrapolated to PEMWE systems, or stabilization effects occur over large timescale operating conditions.

SFC-ICP-MS measurements cannot be carried out for several hours or even days. Thus, electrochemical measurements were

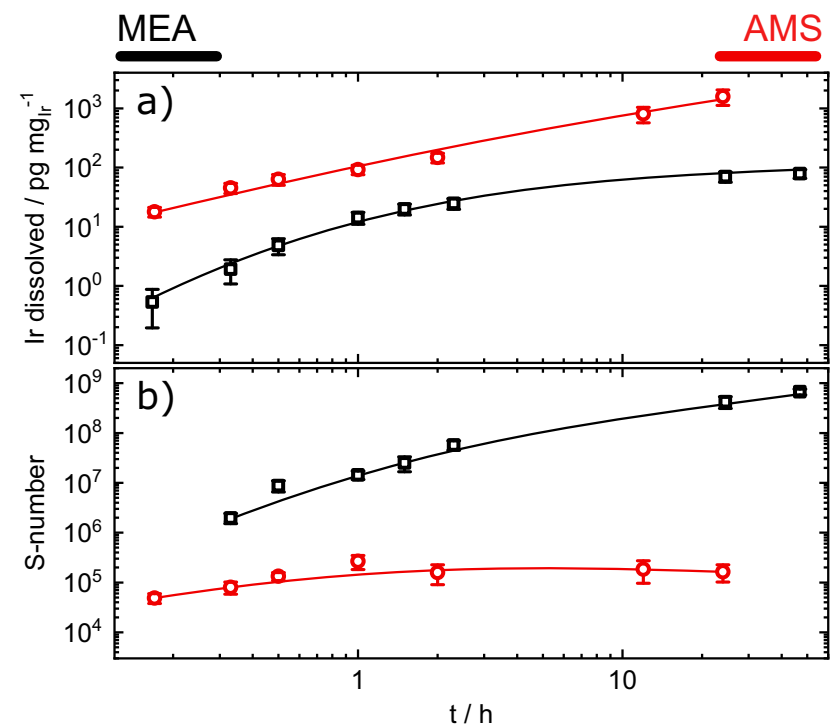

Fig. 5 Long-term stability of IrO $_{x}$ in AMS and MEA environment.

a Loading-normalized total dissolved iridium amount at current densities of $0.2 \mathrm{~A} \mathrm{mg}_{\mathrm{Ir}}{ }^{-1}$ and $2 \mathrm{~A} \mathrm{mg}_{\mathrm{Ir}}{ }^{-1}$ in AMS and MEA, respectively; b S-numbers calculated from the amounts of dissolved iridium. Lines displayed for ease of interpretation. Error bars were calculated as the standard deviation of at least three independent measurements. Source data are provided in the source data file.

carried out in an $\mathrm{H}$-cell configuration to represent an aqueous model system operated at longer timescales. A $0.1 \mathrm{M} \mathrm{H}_{2} \mathrm{SO}_{4}$ electrolyte with sample collection with sample collection from both compartments was used (see experimental). To have a sideby-side comparison, samples from the developed GR-free MEA system were taken from the anode water cycle and the cathode water outlet. Calculations for obtaining mass losses are shown in supplementary note 2 .

Loading-normalized iridium dissolution for $\mathrm{H}$-cell $\left(\mathrm{IrO}_{\mathrm{x}}\right.$ loading $=10 \mu \mathrm{g}_{\mathrm{Ir}} \mathrm{cm}^{-2}$ ) and MEA measurements (anode $\mathrm{IrO}_{\mathrm{x}}$ loading $=1 \mathrm{mg}_{\mathrm{Ir}} \mathrm{cm}^{-2}$ ) are displayed in Fig. 5a. Current densities were $0.2 \mathrm{~A} \mathrm{mg}_{\text {Ir }}{ }^{-1}$ for AMS and $2 \mathrm{~A} \mathrm{mg}_{\text {Ir }}{ }^{-1}$ for MEA. At the employed current density, the amount of dissolved iridium in the aqueous system rose almost constantly throughout the experiment after an early-stage stabilization. S-numbers (Fig. 5b) only stabilized marginally from values of $7 \times 10^{4}$ to $2 \times 10^{5}$. In contrast, the iridium mass loss during DI water-operated MEA experiments stabilized rapidly after the start to an almost constant level. The S-numbers rose from an initial value of $10^{7}$ in the first hours to a value of $10^{8}$ and stabilize after the first day of the experiment at $\sim 10^{9}$ (compare supplementary information 2).

The results obtained in MEA are in heavy contrast with results previously shown by Babic et al. ${ }^{39}$. In their experiment, the authors observed fluctuating or, after an initial increase, decreasing iridium concentrations in the electrolyte. Although a direct protocol comparison is not feasible, a similar decrease of iridium in the water feed was observed by Regmi et al. ${ }^{54}$. Our results clearly show the advantage of the employed metal-free MEA setup, as the authors already pointed out a possible interference of GR in their results. Thus, in all studies concerning dissolution products of operational MEAs, GR should be taken into account, as otherwise, it might result in misleading conclusions.

Previous research would suggest a stabilization under longterm operation on iridium-based catalysts owing to crystallization $^{30}$. Indeed, both aqueous and MEA systems should eventually yield an equivalent degree of stabilization. For MEA 
systems, such stabilization is reached at an earlier stage given the higher operating current density of $2 \mathrm{~A} \mathrm{mg}_{\mathrm{Ir}}{ }^{-1}$ inherent to the system compared with the current density of $0.2 \mathrm{~A} \mathrm{mg}_{\mathrm{Ir}}{ }^{-1}$ employed in our $\mathrm{H}$-cell setup. Because of the low operational currents achieved in AMS, we cannot unambiguously proof such a stabilization effect. A method to circumvent such limitation, beyond the scope of this report, would be to perform studies on high-current density achieving gas diffusion electrode-type (GDE) reactors ${ }^{55-57}$.

System breakdown of the dissolution discrepancy between AMS and MEA. A comparison of these results reveals the inherent differences between MEA and AMS. As shown in Fig. 6, the differences in S-numbers between AMS (1) and MEA decrease to less than one order of magnitude when circulating diluted acid through the MEA water feed (2). These differences cannot be related to a sole factor. Loading, flow rate, dissolved species, timescale or Nafion content, although not relevant factors in AMS, might play a role in MEA and have to be addressed in a follow-up study. However, the higher complexity of the MEA does not allow tangible conclusions on the main contributor. When operating an MEA with DI water on the same timescale (3), the S-number increases by two orders of magnitude, indicating a $\mathrm{pH}$ shift between AMS and MEA as a main contributor to the dissolution discrepancy. After 2 weeks of operation, the Snumber of the MEA increased by more than one order of magnitude (4). At this timescale, based on lifetime calculations from the S-number, the catalyst in the aqueous system would already have been degraded completely. Hence, a stabilization on a larger timescale can be treated as the second main contributor to the dissolution discrepancy. As STEM-energy dispersive X-ray (EDX) only detects small amounts of iridium in the membrane close to the anode catalyst layer, iridium depositing in the membrane can be ruled out as a large contributor.

Given the aforementioned results, we should now address past preconceptions regarding local $\mathrm{pH}$ during PEMWE operation. PEMWE anodes are assumed to operate under highly acidic conditions due to protons generated at the anode side during operation. However, literature is scarce regarding actual $\mathrm{pH}$ value estimation under PEMWE operation. An initial review by Carmo et al. ${ }^{10}$ tentatively estimated a $\mathrm{pH} 2$ value, whereas later investigations reported $\mathrm{pH}$ values in the anode and cathode water cycle of an MEA setup in a range between 5.6 and $3.5^{58}$. However, these results might not be representative for conditions in the anode catalyst layer as the local $\mathrm{pH}$ can decrease owing to proton generation in the water-splitting reaction. The results shown in this study indicate that proton activity in MEA environment is supposedly lower as estimated from the concentrations. Thus, it is likely, that the effective $\mathrm{pH}$ in MEA environment is less acidic as generally accepted in the literature.

These results indicate that future research should emphasize more on two aspects of MEA development: real conditions in the anode catalyst layer and effects in the catalyst/membrane interface.

Conclusions and outlook. In summary, we show that stability measurements performed in AMS have to be treated carefully regarding their relevance for long-term PEMWE applications. The main effects contributing to the dissolution discrepancy were identified as a discrepancy between estimated and real $\mathrm{pH}$ in MEA and stabilization occurring over time.

Based on this research, the community should critically evaluate the process of OER catalyst testing in AMS. Owing to faster degradation, AMS might serve as an ideal system for accelerated stress tests. For this purpose, GDE systems currently under development might help to study OER catalysts in model systems, which resemble the conditions in MEA much better.

However, those results should always be critically compared with experimental MEA data to extract representative conclusions. Furthermore, more focus should be put on the direct evaluation of catalysts under MEA device operation. In particular, a setup consisting of an MEA coupled to downstream analytics would allow a better understanding of dynamic operation conditions, relevant for coupling to renewable energies. We believe that the results shown here will provide improved

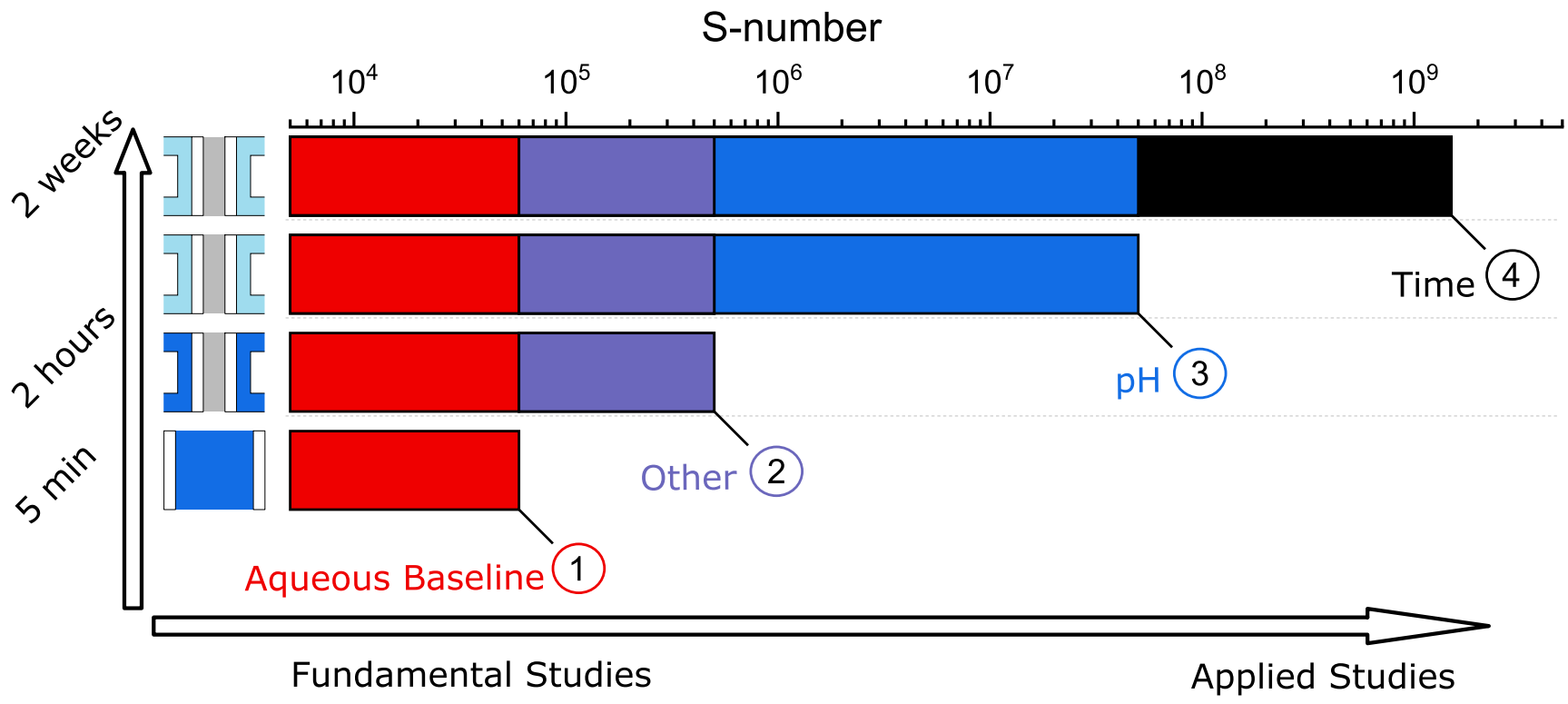

Fig. 6 Scheme on the proposed main contributors to the dissolution discrepancy. Schematic drawing of the factors contributing to the OER catalyst dissolution discrepancies between AMS and MEA. Measurements in AMS exhibit an S-number of $6 \times 10^{4}$ (1). The discrepancy to an MEA operated with acid (2), showing an S-number of $4 \times 10^{5}$, cannot be pointed to a single factor. Various factors such as flow rate, Nafion content, and timescale have to be taken into account. The discrepancy of the MEA operated with acidic water feed to an MEA operated conventionally with DI water (3) of almost two orders of magnitude, however, is related to a $\mathrm{pH}$ shift in the system. With a longer operation time (4), additional stabilization effects in MEA take place. Source data are provided in the source data file. 
guidelines for future catalyst development and testing to mimic realistic MEA operating conditions.

\section{Methods}

\section{SFC-ICP-MS measurements}

Electrode preparation. Electrodes were prepared by suspending commercial Alfa Aesar $\mathrm{IrO}_{\mathrm{x}} \cdot 2 \mathrm{H}_{2} \mathrm{O}$ Premion catalyst powder in a mixture of $87.5 \%$ ultrapure water (Merck Milli-Q), $12.5 \%$ IPA, and Nafion ${ }^{\circledR}$ perfluorinated resin solution (Sigma Aldrich, $5 \mathrm{wt} \%)$. Standard inks had an iridium concentration of $663 \mu \mathrm{gl}^{-1}$, a Nafion concentration of $332 \mu \mathrm{g} \mathrm{l}^{-1}$ and a volume of $1 \mathrm{ml}$ in a $1.5 \mathrm{ml}$ Eppendorf tube. Inks were sonicated for $10 \mathrm{~min}$ ( $4 \mathrm{~s}$ pulse, $2 \mathrm{~s}$ pause) and dropcasted as $0.2 \mu \mathrm{l}$ on a freshly polished glassy carbon plate (SIGRADUR G, HTW). The quality and diameter of the dropcasted catalyst spots ( $\varnothing$ of ca. $1.3 \mathrm{~mm}$ ) was screened by employing Keyence VK-X250 profilometer.

For variations of loading and Nafion content, the concentration of iridium and Nafion in the ink was adjusted to the desired loading and concentration. An IPA: DI water ratio of 12.5:87.5, was employed in all $\mathrm{IrO}_{\mathrm{x}}$ inks, accounting for the alcohol content in the Nafion solution.

Electrochemical measurements. Electrochemical measurements were carried out with an SFC-ICP-MS ${ }^{29}$, with the modifications described in ref. 28 in Ar-saturated $0.1 \mathrm{M} \mathrm{H}_{2} \mathrm{SO}_{4}$ (Merck Suprapur) mixed with ultrapure water. The dropcasted spots, acting here as working electrodes, were located with a top view camera to enable vertical alignment with the SFC $(\varnothing 2 \mathrm{~mm})$. A graphite rod served as counter electrode, whereas a saturated $\mathrm{Ag} / \mathrm{AgCl}$ electrode (Metrohm) was used as reference electrode. ICP-MS measurements were performed with a NexIon 300 spectrometer (Perkin Elmer), employing a flow rate of $208 \mu \mathrm{min}^{-1}$ for reference measurements. For the flow rate-dependence studies, flow rates were adjusted by tuning the speed of the ICP-MS peristaltic pump. Daily calibration of the ICP-MS was performed by freshly prepared standard solutions containing $\operatorname{Ir}\left(0.5\right.$ to $\left.5 \mu \mathrm{g} \mathrm{l}^{-1}\right)$, and $\operatorname{Re}$ $\left(10 \mu \mathrm{g} \mathrm{l}^{-1}\right)$ as an internal standard. All current and dissolution rates shown in this report have been normalized to the nominal loading of the spots.

For the variation of dissolved iridium in the electrolyte, iridium was electrochemically dissolved in $0.1 \mathrm{M} \mathrm{H}_{2} \mathrm{SO}_{4}$ by 1000 cyclic voltammograms recorded in a potential range from $0.05 \mathrm{~V}_{\mathrm{RHE}}-1.5 \mathrm{~V}_{\mathrm{RHE}}{ }^{37,47}$. The iridium concentration was then determined by ICP-MS. Electrolyte and standards were prepared from the electrolyte with dissolved iridium. A baseline measurement was taken before cell contact.

For the variation of $\mathrm{pH}$, the electrolyte was set to the corresponding $\mathrm{H}_{2} \mathrm{SO}_{4}$ concentration. To ensure electronic conductivity of the electrolyte in measurements with a $\mathrm{pH}$ higher than 1 , the total concentration of sulfate ions was set to $0.05 \mathrm{M}$ with $\mathrm{K}_{2} \mathrm{SO}_{4}$ (99.999\% purity, Sigma Aldrich).

\section{H-cell measurements}

Electrode preparation. Electrodes were prepared from Alfa Aesar $\mathrm{IrO}_{\mathrm{x}} \cdot 2 \mathrm{H}_{2} \mathrm{O}$ Premion powder. Ink for electrodes was prepared with ultrapure water (Merck Milli-Q) at a concentration of $283 \mu \mathrm{g} \mathrm{l}^{-1}$ with a volume of $1 \mathrm{ml}$ in an Eppendorf tube. The ink was sonicated for $15 \mathrm{~min}$ ( $4 \mathrm{~s}$ pulse, $2 \mathrm{~s}$ pause) and dropcasted as $10 \mu \mathrm{l}$ on a freshly cleaned FTO plate, previously sonicated for 10 min sequentially in 2\% Hellmanex III (Hellma Analytics) solution, DI water, and ethanol, respectively. The resulting dropcasted Ir catalyst exhibited a diameter of $6 \mathrm{~mm}$ and a loading of $10 \mu \mathrm{g} \mathrm{cm}^{-2}$.

Electrochemical measurements. Electrochemical bulk measurements were carried out in a homemade $\mathrm{H}$-cell. Each compartment was filled with $28 \mathrm{ml} 0.1 \mathrm{M} \mathrm{H}_{2} \mathrm{SO}_{4}$ (Merck Suprapur diluted with Merck Milli-Q) before the experiment. The working electrodes and reference electrodes (Basi, $3 \mathrm{M} \mathrm{Ag} / \mathrm{AgCl}$ ) were immersed in one compartment whereas the counter electrodes (glassy carbon, SIGRADUR G, HTW) were immersed in the other compartment. The compartments were covered with Parafilm to avoid evaporation of electrolytes. Convection in the system for equal distribution of dissolution product was enabled through Ar-purging of the anode compartments. Samples were taken by an automated liquid handler (Gilson GX-271). The electrochemical protocol (Gamry Interface1000 B) was started after the first sample was extracted. The total volume of electrolyte in both compartments was kept between the initial $28 \mathrm{ml}$ and $24 \mathrm{ml}$ at any time.

\section{MEA measurements}

$M E A$ preparation. For the experiments with the PEMWE setup, square format $5 \mathrm{~cm}^{2}$ active cell area MEAs were prepared by a decal transfer method. As catalyst for the OER at the anode side, the same Alfa Aesar $\mathrm{IrO}_{\mathrm{x}} 2 \mathrm{H}_{2} \mathrm{O}$ Premion powder was applied as in the SFC and $\mathrm{H}$-Cell experiments. The anode catalyst loading was $1.03 \pm 0.07 \mathrm{mg}_{\mathrm{Ir}} \mathrm{cm}^{-2}$ for all tests. For the hydrogen evolution reaction at the cathode side, carbon-supported (Vulcan XC72) platinum nanoparticles catalyst (45.8 wt\% Pt/C; TEC10V50E from Tanaka, Japan) with loadings of $0.30 \pm$ $0.14 \mathrm{mg}_{\mathrm{Pt}} \mathrm{cm}^{-2}$ was used. To prepare the catalyst inks, catalyst powder, 2-propanol (purity $\geq 99.9 \%$ from Sigma Aldrich, Germany), and Nafion ${ }^{\circledR}$ ionomer solution ( $20 \mathrm{wt} \%$ ionomer; D2021 from IonPower, USA) are mixed for $24 \mathrm{~h}$ using a roller mill and $5 \mathrm{~mm}$ zirconia grinding balls. The decals were coated with a Mayer-rod coating machine on $50 \mu \mathrm{m}$ PTFE foil (from Angst+Pfister, Germany). Finally, MEAs were hot-pressed $\left(3 \mathrm{~min}\right.$ at $\left.155^{\circ} \mathrm{C}, 2.5 \mathrm{MPa}\right)$ using the decals and different
Nafion ${ }^{\circledast}$ membrane types 117 (180 $\mu$ m thickness), $212(50 \mu \mathrm{m}$ thickness) and 211 (25 $\mu \mathrm{m}$ thickness). By evaluating the weight differences $( \pm 15 \mu \mathrm{g}$; XPE105DR microbalance from Mettler Toledo, Germany) of the PTFE decals before and after hot pressing, the individual catalyst loadings of the MEAs were calculated. All anodes have an ionomer content of $9 \mathrm{wt} \%$, whereas all cathodes have an ionomer to carbon mass ratio of $0.6 / 1$.

MEA measurements. To prevent any precipitation of dissolved Iridium in the cell or in the test rig a special PEMWE setup was developed.

Cell. The cell uses a two-piece monopolar plate concept consisting of a metal flowfield sheet and a plastic body. The flowfield plates are made from $3 \mathrm{~mm}$ grade two titanium sheet with laser cut single serpentine channel (equal $1 \mathrm{~mm}$ land and $1 \mathrm{~mm}$ channel spacing). To prevent galvanic plating of iridium, the titanium flowfield plates are gold-coated $(0.5 \mu \mathrm{m}$ by physical vapor deposition and another $5 \mu \mathrm{m}$ galvanic coating on top). Finally, the metal flowfield plates are inserted into a fitted plastic body made from polyoxymethylene (aqueous tests) or polytetrafluoroethylene (second design for aqueous and diluted sulfuric acid tests). The plastic body allows for media transport from the serpentine flowfield inside the cell without contact to metal surfaces to the in- and outlet fittings made from polypropylene (PP) at the face sides of the monopolar plates. At the anode side, an expanded titanium metal sheet ( $250 \mu \mathrm{m}$ thickness, Sylatech, Germany) with $5 \mu \mathrm{m}$ platinum coating is used as a porous transport layer between MEA and flowfield. The cathode side PTL is a carbon fiber paper (TGP-H-120 from Toray, Japan, no MPL) with a thickness of $370 \mu \mathrm{m}$.

Test rig. A fully automated test rig (E40 by Greenlight Innovation, Canada) equipped with a potentiostat and a booster (Reference 3000 and $30 \mathrm{~A}$ booster, Gamry, USA) was used as the basis for the integration of a metal-free anode water cycle and cathode exhaust water collector. Borosilicate glass bottles of 0.51 to 11 volume were used as the anode water cycle setup tank. A membrane pump (NF30 from KNF, Germany) and PE/PTFE tubes were used to feed the cell with water at a rate of $\sim 300 \mathrm{ml} \mathrm{min}^{-1}$. To maintain an elevated temperature of $\sim 55-60{ }^{\circ} \mathrm{C}$ in the cell, the setup tank glass bottle is tempered by a heater plate (IKA, Germany). To initially clean the setup from ionic impurities a deionizer cartridge is used (Leycopure mixed bed resin from Leyco, Germany), which is bypassed during the actual dissolution experiment. The anode water samples were tapped from the cycle directly behind the cell. As there was no water cycling at the cathode side, the cathode water samples were taken from the PP cathode exhaust water collector bottle. Its reservoir volume was $\sim 10 \mathrm{ml}$ and was continuously flushed at the cathode water exhaust rate of $8.2 \pm 0.4 \mathrm{ml} \mathrm{h}^{-1}$.

Measurement procedure. Before starting the dissolution tests, the anode water cycle is cleaned up at elevated temperature for at least $12 \mathrm{~h}$ by running the water through the deionizer cartridge to remove eventual ionic impurities released from the setup (feed water processed by ULTRA CLEAR ${ }^{\circledast}$ TP ultrapure water system from Evoqua, USA). After the cleaning period, the cartridge is bypassed and the first $10 \mathrm{ml}$ water sample (standard volume for all samples) is tapped from the cycle without contact to the cell as a clean reference. In the next step, the cell is mounted and water is run through the cell for $10 \mathrm{~min}$ without current. At the end, again $10 \mathrm{ml}$ water is tapped from the cycle. Subsequently, the current density was set to $0.2 \mathrm{~A} \mathrm{~cm}^{-2}$ for $10 \mathrm{~min}$ and another water sample was tapped. Thereafter, the current is set to $2 \mathrm{~A} \mathrm{~cm}^{-2}$ where it stays for the rest of the experiment. Anode water samples are tapped after holding intervals of $10 \mathrm{~min}, 30 \mathrm{~min}, 1 \mathrm{~h}, 1 \mathrm{~h}$, and successive every $24 \mathrm{~h}$. Cathode samples are taken for the first time $3 \mathrm{~h}$ after the start of the test and then also every $24 \mathrm{~h}$. During the aqueous tests with fixed anode side water volume the tapped water, the consumed water, and the water lost to the cathode by electroosmosis is replaced by ultrapure feed water. In contrast, during the acidic tests and the aqueous tests with variable water volume, the tapped, consumed or electroosmotically transported electrolyte volume is not replaced and the initial volume of water or $0.1 \mathrm{M} \mathrm{H}_{2} \mathrm{SO}_{4}$ is gradually reduced. At the end of the dissolution test, both flowfields were purged with nitrogen and the cell was held for at least $12 \mathrm{~h}$ at $1.2 \mathrm{~V}$ cell voltage to avoid anode side catalyst reduction by permeating hydrogen from the cathode side or the membrane. In addition, for the last two tests with gradually reduced water/acid volume, polarization curves and electrical impedance spectroscopy were made to reveal the end-of life performance of the aged MEAs. The MEAs are extracted in a glove bag and stored under nitrogen until spectroscopic analysis/STEM graphs were made.

\section{Scanning transmission electron microscopy}

Sample preparation. Samples were embedded in Araldite 502 epoxy resin and cured overnight at $60^{\circ} \mathrm{C}$. Ultrathin sections with a nominal thickness of $100 \mathrm{~nm}$ were cut with an RMC Boeckeler PowerTome using a Diatome ultra $45^{\circ}$ diamond knife. The sections were collected on copper grids for subsequent imaging via scanning transmission electron microscopy.

Measurements. STEM micrographs were taken with a Zeiss Crossbeam 540 FIBSEM with annular STEM with a detector accelerating voltage of $20 \mathrm{kV}$ and probe current of $300 \mathrm{pA}$. High-angle annular dark-field was chosen as imaging mode, 
owing to its high contrast between atoms with low atomic number $\mathrm{Z}$ (membrane: $\mathrm{F}, \mathrm{C}, .$. ) and a high atomic number ( $\mathrm{Ir}, \mathrm{Au}, \mathrm{Ti})$. The chemical composition was determined via EDX (X-Max 150 silicon drift detector, Oxford Instruments; Software: Aztec Version 3.3, Oxford instruments) with an accelerating voltage of $20 \mathrm{kV}$ and a probe current of $300 \mathrm{pA}$.

STEM micrographs were post-processed with ImageJ. As the samples have an average thickness of $100 \mathrm{~nm}$ while the penetrating depth of the electron beam can be several micrometers, EDX analysis contains background elemental information like copper from the TEM-grid. For reasons of simplification, only elements of interest $(\mathrm{Pt}, \mathrm{Ir}, \mathrm{Au}, \mathrm{Ti}, \mathrm{C}, \mathrm{F}$, and $\mathrm{O})$ are shown in the elemental point measurements.

\section{Data availability}

The authors declare that the main data supporting the findings of this study are available within the article and its Supplementary Information. Source data are provided with this paper. Extra data are available from the corresponding authors upon reasonable request. Source data are provided with this paper.

Received: 13 December 2020; Accepted: 9 March 2021; Published online: 13 April 2021

\section{References}

1. De Luna,P., et al. What would it take for renewably powered electrosynthesis to displace petrochemical processes? Science 364, eaav3506 (2019).

2. Bockris, J. O. A hydrogen economy. Science 176, 1323 (1972).

3. Staffell, I. et al. The role of hydrogen and fuel cells in the global energy system. Energy Environ. Sci. 12, 463-491 (2019).

4. Ardo, S. et al. Pathways to electrochemical solar-hydrogen technologies. Energy Environ. Sci. 11, 2768-2783 (2018).

5. Bareiß, K. et al. Life cycle assessment of hydrogen from proton exchange membrane water electrolysis in future energy systems. Appl. Energy 237, 862-872 (2019).

6. Kopernikus P2X Project funded by the German Ministry of Education and Research (BMBF). [https://www.kopernikus-projekte.de/projekte/p2x] 2020 [cited 2020 June 19th].

7. Stockford, C. et al. H2FC SUPERGEN: An overview of the Hydrogen and Fuel Cell research across the UK. Int. J. Hydrog. Energy 40, 5534-5543 (2015).

8. Miller, H. A., et al. Green hydrogen from anion exchange membrane water electrolysis: a review of recent developments in critical materials and operating conditions. Sustainable Energ. Fuels https://doi.org/10.1039/C9SE01240K (2020).

9. Abbasi, R. et al. A Roadmap to Low-Cost Hydrogen with Hydroxide Exchange Membrane Electrolyzers. Adv. Mater. 31, e1805876 (2019).

10. Carmo, M. et al. A comprehensive review on PEM water electrolysis. Int. J. Hydrog. Energy 38, 4901-4934 (2013).

11. Schmidt, O. et al. Future cost and performance of water electrolysis: An expert elicitation study. Int. J. Hydrog. Energy 42, 30470-30492 (2017).

12. Ayers, K. E. et al. Pathways to ultra-low platinum group metal catalyst loading in proton exchange membrane electrolyzers. Catal. Today 262, 121-132 (2016).

13. Bernt, M., Siebel, A. \& Gasteiger, H. A. Analysis of voltage losses in PEM water electrolyzers with low platinum group metal loadings. J. Electrochem. Soc. 165, F305-F314 (2018).

14. Ayers, K. E., L. T. Dalton, and E. B. Anderson. Efficient generation of high energy density fuel from water. 41, 27 (2012).

15. Spoeri, C., et al. Experimental activity descriptors for iridium-based catalysts for the electrochemical oxygen evolution reaction (OER). ACS Catal. 10, 14640-14648 (2020).

16. Ledendecker, M., et al. Towards maximized utilization of iridium for the acidic oxygen evolution reaction. Nano Res. 12, 2275-2280 (2019).

17. Hufnagel, A. G. et al. Carbon-templated conductive oxide supports for oxygen evolution catalysis. Nanoscale 11, 14285-14293 (2019).

18. Böhm, D., et al. Efficient OER catalyst with low Ir volume density obtained by homogeneous deposition of iridium oxide nanoparticles on macroporous antimony-doped tin oxide support. Adv. Funct. Mater. 30, 1906670 (2020).

19. Seitz, L. C. et al. A highly active and stable $\mathrm{IrOx} / \mathrm{SrIrO} 3$ catalyst for the oxygen evolution reaction. Science 353, 1011-1014 (2016).

20. Diaz-Morales, O. et al. Iridium-based double perovskites for efficient water oxidation in acid media. Nat. Commun. 7, 12363 (2016).

21. Nong, H. N. et al. Oxide-supported $\mathrm{IrNiO}(\mathrm{x})$ core-shell particles as efficient, cost-effective, and stable catalysts for electrochemical water splitting. Angew. Chem. Int. Ed. Engl. 54, 2975-2979 (2015).

22. Strickler, A. L., et al. Systematic investigation of iridium-based bimetallic thin film catalysts for the oxygen evolution reaction in acidic media. ACS Appl. Mater. Interfaces 37, 34059-34066 (2019).
23. Binninger, T. et al. Thermodynamic explanation of the universal correlation between oxygen evolution activity and corrosion of oxide catalysts. Sci. Rep. $\mathbf{5}$, 12167 (2015)

24. Schmidt, T. J. et al. Characterization of high-surface area electrocatalysts using a rotating disk electrode configuration. J. Electrochem. Soc. 145, 2354-2358 (1998).

25. Mayrhofer, K. J. J. et al. Measurement of oxygen reduction activities via the rotating disc electrode method: From Pt model surfaces to carbon-supported high surface area catalysts. Electrochim. Acta 53, 3181-3188 (2008).

26. Garsany, Y. et al. Experimental methods for quantifying the activity of platinum electrocatalysts for the oxygen reduction reaction. Anal. Chem. 82, 6321-6328 (2010)

27. Frydendal, R. et al. Benchmarking the stability of oxygen evolution reaction catalysts: the importance of monitoring mass losses. ChemElectroChem $\mathbf{1}$, 2075-2081 (2014).

28. Geiger, S. et al. The stability number as a metric for electrocatalyst stability benchmarking. Nat. Catal. 1, 508-515 (2018).

29. Klemm, S. O. et al. Coupling of a high throughput microelectrochemical cell with online multielemental trace analysis by ICP-MS. Electrochem. Commun. 13, 1533-1535 (2011).

30. Li, T. et al. Atomic-scale insights into surface species of electrocatalysts in three dimensions. Nat. Catal. 1, 300-305 (2018).

31. Cherevko, S. Stability and dissolution of electrocatalysts: building the bridge between model and "real world" systems. Curr. Opin. Electrochem. 8, 118-125 (2018).

32. Alia, S. M. et al. Activity and durability of iridium nanoparticles in the oxygen evolution reaction. J. Electrochem. Soc. 163, F3105-F3112 (2016).

33. Alia, S. M. \& Anderson, G. C. Iridium oxygen evolution activity and durability baselines in rotating disk electrode half-cells. J. Electrochem. Soc. 166, F282-F294 (2019)

34. Kim, Y. T. et al. Balancing activity, stability and conductivity of nanoporous core-shell iridium/iridium oxide oxygen evolution catalysts. Nat. Commun. 8, 1449 (2017).

35. Cherevko, S. et al. Oxygen and hydrogen evolution reactions on $\mathrm{Ru}, \mathrm{RuO} 2, \mathrm{Ir}$, and $\mathrm{IrO} 2$ thin film electrodes in acidic and alkaline electrolytes: A comparative study on activity and stability. Catal. Today 262, 170-180 (2016).

36. Cherevko, S. et al. Oxygen evolution activity and stability of iridium in acidic media. Part 1. - metallic iridium. J. Electroanal. Chem. 773, 69-78 (2016).

37. Cherevko, S. et al. Oxygen evolution activity and stability of iridium in acidic media. Part 2. - electrochemically grown hydrous iridium oxide. J. Electroanal. Chem. 774, 102-110 (2016).

38. Siracusano, S. et al. New insights into the stability of a high performance nanostructured catalyst for sustainable water electrolysis. Nano Energy $\mathbf{4 0}$ 618-632 (2017).

39. Babic, U., et al. Understanding the effects of material properties and operating conditions on component aging in polymer electrolyte water electrolyzers. $J$. Power Sources 451, 227778 (2020).

40. Onda, K., et al. Performance analysis of polymer-electrolyte water electrolysis cell at a small-unit test cell and performance prediction of large stacked cell. $J$. Electrochem. Soc. 149, A1069 (2002)

41. Shin, H.-S. \& Oh, B. S. Water transport according to temperature and current in PEM water electrolyzer. Int. J. Hydrog. Energy 45, 56-63 (2020).

42. Arminio-Ravelo, J. A., et al. Electrolyte effects on the electrocatalytic performance of iridium-based nanoparticles for oxygen evolution in rotating disc electrodes. Chemphyschem 20, 2956-2963 (2020).

43. Pham, C. V., et al. IrO2 coated $\mathrm{TiO} 2$ core-shell microparticles advance performance of low loading proton exchange membrane water electrolyzers. Appl. Catal. B: Environ. 269, 118762 (2020).

44. da Silva, G. C. et al. Dissolution stability: the major challenge in the regenerative fuel cells bifunctional catalysis. J. Electrochem. Soc. 165 F1376-F1384 (2018).

45. Silva, G. C. et al. Oxygen evolution reaction on tin oxides supported iridium catalysts: do we need dopants? ChemElectroChem 7, 2330-2339 (2020).

46. Rozain, C. et al. Influence of iridium oxide loadings on the performance of PEM water electrolysis cellsart II - advanced oxygen electrodes. Appl. Catal. B: Environ. 182, 123-131 (2016).

47. Pickup, P. G. \& Birss, V. I. A model for anodic hydrous oxide growth at iridium. J. Electroanal. Chem. Interf. Electrochem. 220, 83-100 (1987).

48. Giordano, L. et al. $\mathrm{pH}$ dependence of OER activity of oxides: current and future perspectives. Catal. Today 262, 2-10 (2016).

49. Lædre, S. et al. Materials for proton exchange membrane water electrolyzer bipolar plates. Int. J. Hydrog. Energy 42, 2713-2723 (2017).

50. Hsueh, K.-L., et al. Direct methanol fuel cells. Electrochemical Technologies for Energy Storage and Conversion. 701-727 (2012).

51. Naito, T., et al. Water electrolysis in saturated phosphate buffer at neutral $\mathrm{pH}$. ChemSusChem 13, 5921-5933 (2020).

52. Rakousky, C. et al. The stability challenge on the pathway to high-currentdensity polymer electrolyte membrane water electrolyzers. Electrochim. Acta 278, 324-331 (2018). 
53. Kopp, M. et al. Energiepark Mainz: Technical and economic analysis of the worldwide largest Power-to-Gas plant with PEM electrolysis. Int. J. Hydrog. Energy 42, 13311-13320 (2017).

54. Regmi, Y. N., et al. A low temperature unitized regenerative fuel cell realizing 60\% round trip efficiency and 10000 cycles of durability for energy storage applications. Energy Environ. Sci. https://doi.org/10.1039/D0EE02970J (2020).

55. Pinaud, B. A. et al. Key Considerations for High Current Fuel Cell Catalyst Testing in an Electrochemical Half-Cell. J. Electrochem. Soc. 164, F321-F327 (2017).

56. Inaba, M. et al. Benchmarking high surface area electrocatalysts in a gas diffusion electrode: measurement of oxygen reduction activities under realistic conditions. Energy Environ. Sci. 11, 988-994 (2018).

57. Ehelebe, K. et al. Evaluating Electrocatalysts at Relevant Currents in a Half Cell: The Impact of Pt Loading on Oxygen Reduction Reaction. J. Electrochem. Soc. 166, F1259-F1268 (2019).

58. Langemann, M. et al. Validation and characterization of suitable materials for bipolar plates in PEM water electrolysis. Int. J. Hydrog. Energy 40, 11385-11391 (2015)

\section{Acknowledgements}

J.K., M.M, M.R., and S.C. acknowledge funding by the German Federal Ministry of Education and Research (BMBF) within the Kopernikus Project P2X under the grant number 03SFK211-2. D.E.-L. and S.C. acknowledge financial support from Deutsche Forschungsgemeinschaft DFG under project number $\mathrm{CH}$ 1763/3-1 and 1763/4-1. We thank the chair of Technical Electrochemistry at the Technical University of Munich for providing the MEA preparation equipment. We thank Carina Schramm for electrode preparation.

\section{Author contributions}

S.C. conceived and developed the idea and coordinated the work. J.K. and S.C. designed the experiments. J.K. prepared electrodes for aqueous measurements, performed dissolution measurements in aqueous electrolyte, liquid sample analysis, and data analysis. M.M. developed the GR-free MEA setup, prepared MEAs, performed MEA measurements, and data analysis. K.S. performed H-cell measurements. M.B. performed STEM measurements. T.B. performed ultramicrotome thin-film cuts for STEM. The manuscript was written by J.K., M.M., D. E.-L., and S.C. with input and feedback from all authors.
All authors contributed through scientific discussions and have given approval to the final version of the manuscript.

\section{Funding}

Open Access funding enabled and organized by Projekt DEAL.

\section{Competing interests}

The authors declare no competing interests.

\section{Additional information}

Supplementary information The online version contains supplementary material available at https://doi.org/10.1038/s41467-021-22296-9.

Correspondence and requests for materials should be addressed to J.K. or S.C.

Peer review information Nature Communications thanks the anonymous reviewers for their contributions to the peer review of this work. Peer review reports are available.

Reprints and permission information is available at http://www.nature.com/reprints

Publisher's note Springer Nature remains neutral with regard to jurisdictional claims in published maps and institutional affiliations.

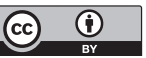

Open Access This article is licensed under a Creative Commons Attribution 4.0 International License, which permits use, sharing, adaptation, distribution and reproduction in any medium or format, as long as you give appropriate credit to the original author(s) and the source, provide a link to the Creative Commons license, and indicate if changes were made. The images or other third party material in this article are included in the article's Creative Commons license, unless indicated otherwise in a credit line to the material. If material is not included in the article's Creative Commons license and your intended use is not permitted by statutory regulation or exceeds the permitted use, you will need to obtain permission directly from the copyright holder. To view a copy of this license, visit http://creativecommons.org/ licenses/by/4.0/.

(C) The Author(s) 2021 